\title{
5.7 ROTATIONAL BURSTING OF INTERPLANETARY DUST PARTICLES
}

\author{
Stephen J. Paddack \\ Goddard Space Flight Center, Greenbelt, Maryland 20771 \\ John W. Rhee \\ (On sabbatical leave from Rose-Hulman Institute of Technology, Terre Haute, Indiana 47803) \\ Goddard Space Flight Center, Greenbelt, Maryland 20771 \\ and \\ University of Maryland, College Park, Maryland 20740
}

Abstract. Solar radiation pressure can cause rotational bursting and eventual elimination from the solar system of small asymmetric interplanetary particles by a windmill effect. The life span determined by this process for stony meteoritic material or tektite glass with radii of $1 \mathrm{~cm}$ is on the order of $10^{5}$ years. Same size material which contains iron, nickel or aluminum, with properties such that it is subject to 5 pe rcent of the amount of spin damping as pure metals, can be removed from the solar system on the order of $10^{6}$ years by this process. Ordinary chondritic material, despite its high resistivity, is subject to a type of magnetic spin damping, in addition to the normal spin damping, with the consequent result that this type material cannot be removed from the solar system by this process. This depletion mechanism appears to work faster than the traditional Poynting-Robertson effect by approximately two orders of magnitude for the nonmetallic particles and one order of magnitude for the metallic particles.

Introduction. Kresák (1968) has drawn attention to a puzzling deficiency in the numbers of small meteors. From the laws of celestial mechanics he notes that the breakup time of meteor showers is on the order of $10^{3}$ to $10^{5}$ years. After the breakup of the showers, the meteors continue to exist as individuals in individual orbits (sporadic meteors) until they are destroyed. Because there are only about five times as many sporadic meteors as shower meteors, the lifetime of meteors as solid objects must have a range of $5 \times 10^{3}$ to $5 \times 10^{5}$ years. But lifetime determined by the Poynting-Robertson effect is about $10^{7}$ years for a $1 \mathrm{~cm}$ radius particle. Hence, some other more effective mechanism of destruction must exist. We here suggest that it is rotational bursting.

Radzievskii (1954) suggested that solar radiation pressure could cause rotational bursting of asteroids and meteoroids having irregular albedos over their entire surfaces. As an example, he calculated that a $1 \mathrm{~cm}$ granite cube at 0.4 au from the sun would reach bursting speed in about 1000 years.

Paddack $(1969,1973)$ demonstrated that rotational bursting could be caused by the interaction of solar radiation pressure and irregular surface geometry irrespective of variations in albedo. In rotational bursting, smaller particles are produced with each burst. Subsequently, they will reach a size where the gravitational attraction to the sun is balanced by the repelling 
effect of radiation pressure also some particles will reach hyperbolic speeds. Once this point is reached, the particles can no longer remain in the solar system and are removed from it.

On the basis of experimentation, it was conservatively estimated that nonmagnetic meteoroids and tektites in heliocentric orbits reach bursting speed in about 60,000 years. These analyses did not take into account magnetic spin damping effects for tektites because of their high electrical resistivity (Hoyte et al., 1965) and low magnetic permeability. Most meteoritic dust is stony (Öpik, 1958; Banderman, 1968) but also contains a metallic component which may cause them to be subject to spin damping. Some stony meteorites have been found to contain between a few tenths of a percent and 20 percent metal (Wood, 1963). Damping has been investigated since (Paddack and Rhee, 1975) and the following is an extension of that analysis.

Analytical Procedure. An irregularly shaped metallic body can also reach rotational bursting speed by this windmill effect. Applying the experimentally determined asymmetry factor, $\ell$, which is 0.05 percent of the maximum dimension of the body (Paddack, 1969, 1973), and using radiation pressure, $P_{r}$, and total projected area, $A$, the effective torque, $N_{1}$, due to radiation pressure, is given by

$$
\mathrm{N}_{1}=0.1 \mathrm{P}_{\mathrm{r}} \mathrm{Al}
$$

That the angular speed of a conductor is reduced in an external magnetic field is a wellknown phenomenon. The magnitude of the retarding torque for a rotating sphere having angular velocity $\omega$ and effective radius $b$ is given by Wilson (1961).

$$
\mathrm{N}_{2}=-\frac{2 \pi \mathrm{B}^{2} \mathrm{~b}^{5} \omega}{15 \rho}
$$

where $\rho$ is the resistivity and B is the interplanetary magnetic field intensity.

For material with randomly distributed metal particles, such as exhibited by some chondrites (Wood, 1963), there is a magnetic spin damping effect related to, and in addition to, normal magnetic spin damping. In this case, each small particle of metal imbedded in the rotating small celestial body tends to retard its spin. The intergrated effect of these metallic particles can cause significant spin damping. The imbedded metal particle torque, $\mathrm{N}_{3}$, is given by

$$
N_{3}=-\left[\frac{2 \pi B^{2} c^{5} \omega}{15 \rho_{c}}\right] \frac{M}{m_{c}}
$$

where $c$ is the average radius of the imbedded metallic particles, $\rho_{c}$ is the resistivity of the imbedded metallic particles, $M$ is the total mass of the metal in the small celestial body, and $m_{c}$ is mass of an average single imbedded metallic particle. 
Damping due to induced currents was also considered but it was found to be insignificantly small. Consequently, it is possible to express the rotational equation of motion for small interplanetary particles, including the damping effects due to interplanetary magnetic field, as

$$
\mathrm{I} \frac{\mathrm{d} \omega}{\mathrm{dt}}=\mathrm{N}_{1}+\mathrm{N}_{2}+\mathrm{N}_{3}
$$

where $I$ is the moment of inertia. The solution of Equation 4 is

$$
\omega=\frac{\alpha}{\beta}\left(1-\mathrm{e}^{-\beta \mathrm{t}}\right)
$$

where $\alpha=\mathrm{N}_{1} / \mathrm{I}$, and $\beta=-\left(\mathrm{N}_{2}+\mathrm{N}_{3}\right) / \mathrm{I} \omega$

Equation 5 shows that the angular motion is stabilized under the damping effect and its upper limit is $\alpha / \beta$.

According to Timoshenko (1942), the stress, $\sigma$, developed in a rotating solid disk of radius $b$ is given by

$$
\sigma=\frac{(3+\mu)}{8} \delta b^{2} \omega^{2}
$$

where $\delta$ is the density and $\mu$ is the Poisson ratio. The time needed for elimination from the solar system by radiation pressure as a consequence of successive bursting can be estimated by multiplying the time to the first burst by a factor at 2.7 (Paddack, 1973) .

The time to elimination from the solar system for certain kinds of materials has been calculated by use of Equations 5 and 6 and tabulated in Table 1. The following assumptions were used: heliocentric circular orbits at one astronomical unit, radiation pressure of $4.5 \times 10^{-5} \mathrm{dyn}$ $\mathrm{cm}^{-2}$, 5 gamma for B (the interplanetary field intensity (Ness, 1965)), standard resistivity for the metals, $3 \times 10^{+14} \mathrm{ohm}-\mathrm{cm}$ for tektites (Hoyte et al. , 1965), $15 \mathrm{ohm}-\mathrm{cm}$ for meteoroids (Wood, 1963), and velocity of $30 \mathrm{~km} \mathrm{sec}-1$. Handbook values were used for the tensile strength

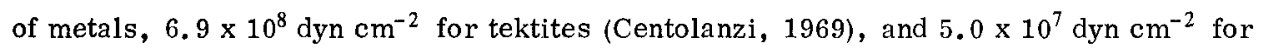
meteoroids (Öpik, 1958). Handbook values were used for the densities of metals, $2.4 \mathrm{~g} \mathrm{~cm}^{-3}$ for tektites (Chao, 1963), and $3.6 \mathrm{~g} \mathrm{~cm}^{-3}$ for meteoroids (Wood, 1963). In the same table, those particles that reach equilibrium below bursting angular speed are indicated as NB (no bursting).

In the case of chondritic meteoroids, metallic particles are assumed to be randomly distributed in them. These imbedded metallic particles are assumed to be iron spherules with radii varying from 25 to $250 \mu \mathrm{m}$. The percentage of iron in the chondrites was varied from 0.5 to 5 percent (Wood, 1963). For the only elimination time shown in the table (actually time to equilibrium in this case), a percentage of iron of 4 percent was assumed, and the radii of the imbedded particles was assumed to be $25 \mu \mathrm{m}$. 
Table 1

Elimination Time for Different Materials as a Function of Particle Radius

\begin{tabular}{|c|c|c|c|c|c|c|c|c|c|}
\hline \multirow{2}{*}{$\begin{array}{l}\text { Radius } \\
\text { (cm) }\end{array}$} & \multicolumn{2}{|c|}{$\mathrm{Al}$} & \multicolumn{2}{|c|}{$\mathrm{Fe}$} & \multicolumn{2}{|c|}{$\mathrm{Ni}$} & \multirow{2}{*}{ Tektites } & \multirow{2}{*}{$\begin{array}{l}\text { Chondritic } \\
\text { Meteoroids }\end{array}$} & \multirow{2}{*}{$\begin{array}{l}\text { Stony Meteor } \\
\text { (nonmetallic) }\end{array}$} \\
\hline & $100 \%$ & $5 \%$ & $100 \%$ & $5 \%$ & $100 \%$ & $5 \%$ & & & \\
\hline $10^{-4}$ & $1.52 \times 10^{2}$ & $1.52 \times 10^{2}$ & $6.25 \times 10^{2}$ & $6.25 \times 10^{2}$ & $1.49 \times 10^{2}$ & $1.49 \times 10^{2}$ & $6.43 \times 10^{1}$ & $* *$ & $2.12 \times 10^{1}$ \\
\hline $10^{-3}$ & $1.52 \times 10^{3}$ & $1.52 \times 10^{3}$ & $6.26 \times 10^{3}$ & $6.25 \times 10^{3}$ & $1.49 \times 10^{3}$ & $1.49 \times 10^{3}$ & $6.43 \times 10^{2}$ & $* *$ & $2.12 \times 10^{2}$ \\
\hline $10^{-2}$ & $1.53 \times 10^{4}$ & $1.52 \times 10^{4}$ & $6.30 \times 10^{4}$ & $6.26 \times 10^{4}$ & $1.50 \times 10^{4}$ & $1.49 \times 10^{4}$ & $6.43 \times 10^{3}$ & NB* & $2.12 \times 10^{3}$ \\
\hline $10^{-1}$ & $1.64 \times 10^{5}$ & $1.52 \times 10^{5}$ & $6.76 \times 10^{5}$ & $6.28 \times 10^{5}$ & $1.51 \times 10^{5}$ & $1.49 \times 10^{5}$ & $6.43 \times 10^{4}$ & NB* & $2.12 \times 10^{4}$ \\
\hline 1 & $\mathrm{NB}^{*}$ & $1.58 \times 10^{6}$ & NB* & $6.49 \times 10^{6}$ & $1.65 \times 10^{6}$ & $1.50 \times 10^{6}$ & $6.43 \times 10^{5}$ & $1.03 \times 10^{6}$ & $2.12 \times 10^{5}$ \\
\hline
\end{tabular}

*NB means no bursting.

**No calculation done because imbedded metallic particle size is equal to or greater than size of small celestial body.

Summary. Solar radiation pressure can cause rotational bursting of certain small asymmetric interplanetary particles and eliminate them from the solar system. Particle lifetimes of between a few tens of years and on the order of $10^{6}$ years a re predicted for particles in heliocentric orbits at $1 \mathrm{au}$. The lifetimes vary as a function of the amount of spin damping to which the particles are subject, their external geometry, their chemical composition, and their physical structure. Particles subject to substantial spin damping, such as some pure metals, or chondritic material with metallic particles imbedded in it, may become stabilized before reaching the bursting point.

For nonmetallic particles, such as tektites or other metal-free silicate material, the retarding torques are so small that there is no effective spin stabilization, and consequently these can be eliminated from the solar system inbetween a few tens of years and in about 60,000 years for particles with radii between $10^{-4}$ and $1 \mathrm{~cm}$ in heliocentric orbits at $1 \mathrm{au}$.

This depletion mechanism works faster than the traditional Poynting-Robertson effect by approximately one order of magnitude for metallic particles, which are not stabilized by spin damping, and about two orders of magnitude for nonmetallic particles.

The percentage of pure nickel-iron-type meteors, which are about gram size, has been found to be only about 1 or 2 percent. Also, observational evidence indicates silicate rather than metallic material for the composition of dust in comets (Millman, 1974). Therefore, a great percentage of the particulate material in interplanetary space should be affected by this process.

For those particles with substantial amounts of metal, the distribution of the metallic component can have a significant effect on spin damping. Any gaps in the conducting path can appreciably lower the effectiveness of normal magnetic spin damping. The spin damping effect of particles of metal imbedded in the small celestial body, however, can be substantial, as was shown for chondritic meteoroids. Despite the spin damping that can occur, rotational bursting 
appears to be a reasonably important process to be considered in the study of the lifetime and population of small bodies in the solar system. It is also reasonable to expect those particles subject to substantial spin damping to be older particles, because they would be eliminated or destroyed by slower acting mechanisms.

Acknowledgments. The authors would like to thank Drs. John A. O'Keefe and David P. Stern for helpful discussions.

\section{References}

Banderman, Lothar W., Physical Properties and Dynamics of Interplanetary Dust, Ph.D Thesis, University of Maryland, 1968, p. 189.

Centolanzi, Frank J., Maximum Tektite Size as Limited by Thermal Stress and Aerodynamic Loads, J. Geophys. Res. 74, pp. 6725-6736, 1969.

Chao, E. C. T., The Petrographic and Chemical Characteristics of Teltites, in Tektites, edited by J. A. O'Keefe, pp. 51-94, University of Chicago Press, Chicago, Ill. 1963.

Hoyte, A., F. Senftle, and P. Wirtz, Electrical Resistivity and Viscosity of Tektite Glass, J. Geophys. Res., 70, pp. 1985-1994, 1965.

Kresák, L.., Structure and Evolution of Meteor Streams, in Physics and Dynamics of Meteors, edited by L. Kresák and P. M. Millman, pp. 391-403, D. Reidel Pub. Co., Dordrecht, Holland, 1968.

Millman, Peter M., Dust in the Solar System, Smithsonian Contributions to Astrophysics, (in press), 1974, pp. 17-18.

Ness, Norman F., The Interplanetary Medium, in Introduction to Space Science, edited by Hess, Wilmot N., pp. 323-346, Gordon and Breach, New York, 1965.

Öpik, Ernst J., Physics of Meteor Flight in the Atmosphere, pp. 24-26, Interscience Pub. Inc., New York, 1958.

Paddack, Stephen J., Rotational Bursting of Small Celestial Bodies: Effects of Radiation Pressure, J. Geophys. Res., 74, pp. 4379-4381, 1969.

Paddack, Stephen J., Rotational Bursting of Small Celestial Bodies: Effects of Radiation Pressure, Ph. D. Thesis, Catholic University of America, Washington, D. C., 1973.

Paddack, Stephen J. and Rhee, John W. , Rotational Bursting of Interplanetary Dust Particles, Geophys. Res. Letters, 2, pp. 365-367, 1975.

Radzievskii, V. V., A Mechanism of the Disintegration of Asteroids and Meteorites, Dokl. Akad. Nauk SSSR, 97, pp. 49-52, 1954.

Timoshenko, S., Strength of Materials, p. 249, D. Van Nostrand, Princeton, N.J., 1942.

Wilson, R. H., Rotational Magnetohydrodynamics and Steering of Space Vehicles, NASA TN 566, Goddard Space Flight Center, 1961.

Wood, J. A., Physics and Chemistry of Meteorites, in The Moon, Meteorites, and Comets,

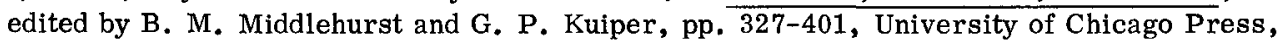
Chicago, Illinois, 1963. 